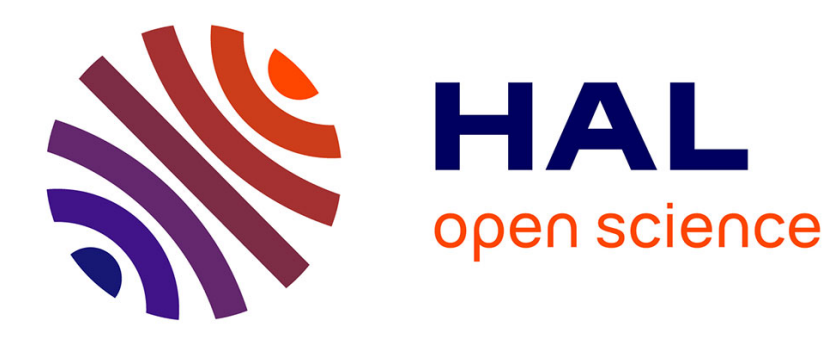

\title{
The screening effect in suspensions of freely moving spheres
}

J. Boissonade

\section{To cite this version:}

J. Boissonade. The screening effect in suspensions of freely moving spheres. Journal de Physique Lettres, 1982, 43 (11), pp.371-375. 10.1051/jphyslet:019820043011037100 . jpa-00232061

\section{HAL Id: jpa-00232061 https://hal.science/jpa-00232061}

Submitted on 1 Jan 1982

HAL is a multi-disciplinary open access archive for the deposit and dissemination of scientific research documents, whether they are published or not. The documents may come from teaching and research institutions in France or abroad, or from public or private research centers.
L'archive ouverte pluridisciplinaire HAL, est destinée au dépôt et à la diffusion de documents scientifiques de niveau recherche, publiés ou non, émanant des établissements d'enseignement et de recherche français ou étrangers, des laboratoires publics ou privés. 


\title{
LE JOURNAL DE PHYSIQUE-LETTRES
}

J. Physique - LETTRES 43 (1982) L- 371 - L-375

1er JUIN 1982, PAGE L-371

Classification

Physics Abstracts

$47.55-82.70$

\section{The screening effect in suspensions of freely moving spheres}

\author{
J. Boissonade $(*)$ \\ Institut für Theoretische Physik, Cologne University, 5000 Köln 41, West Germany
}

(Reçu le 9 février 1982, révisé le 13 avril, accepté le 14 avril 1982)

\begin{abstract}
Résumé. - Le concept d'" effet d'écran " dans les suspensions de sphères libres est précisé. Un problème simple est'proposé pour l'étude de cet effet à la fois d'un point de vue théorique et expérimental. On suggère en outre, sur la base d'hypothèses simples, que la longueur d'écran se comporte comme l'inverse de la fraction volumique dans la limite des fortes dilutions. Des méthodes plus élaborées sont proposées pour vérifier et étendre ce résultat.
\end{abstract}

\begin{abstract}
The signification of the " screening effect " in suspensions of freely moving spheres is discussed. A simple problem is proposed to study this effect from both the theoretical and the experimental points of view. We suggest on the basis of simple assumptions that the predicted screening length behaves as the inverse of the volume fraction in the limit of dilute suspensions. More elaborate methods are proposed to check and extend this result.
\end{abstract}

The rheology of suspensions at high concentrations is one of the main challenge for physicists in fluid mechanics and statistical physics. According to the complexity of the hydrodynamic interactions, it is almost hopeless to expect that the rigorous mathematical techniques which have been successfully applied to low concentrations $[1,2,3]$ could be, in practice, extended to the high concentration domain. One should rather need statistical tools close to those developed in condensed matter theory and statistical theory. Such approaches have been used, for example, by De Gennes [4] who proposed to explain the phenomenon of "plug flows " in the frame of the percolation theory and by Stauffer and Clavin [5] who used a Monte Carlo simulation technique in the sedimentation problem. Here we focus on problems related to the definition of " screening ", which could be used as a starting point for a mean field theory.

(*) Permanent address : Centre de Recherche Paul Pascal, Université de Bordeaux I, Domaine Universitaire, 33405 Talence, France. 
In the sequel we shall limit ourselves to "ideal suspensions " (or " dispersions ") of monodisperse solid spheres free to move in an incompressible fluid, in which the only interactions are hydrodynamical (excluding Brownian motion, Van der Waals or molecular forces, electric or magnetic fields, ...), and in the limit where the size of the spheres and the velocities in the fluid are small enough for the internal forces to be neglected, that is when the Reynolds number $R e$ is small in regard to unity. Let us consider such a suspension in a stationary state, so that the velocity field $\mathbf{v}(\mathbf{r})$ in the fluid phase is the solution of the Navier-Stokes equations [6, 7] with $R e \ll 1$ and non-slip boundary conditions :

$$
\left.\begin{array}{rl}
\eta_{0} \Delta \mathbf{v} & =\operatorname{grad} p \\
\operatorname{div} \mathbf{v} & =0 \\
\mathbf{v} & =\mathbf{v}_{i} \wedge\left(\mathbf{r}-\mathbf{r}_{i}\right), \quad\left|\mathbf{r}-\mathbf{r}_{i}\right|=a
\end{array}\right\}
$$

$\eta_{0}$ is the viscosity of the pure fluid phase, $\mathbf{v}_{i}$ the velocity of the sphere centred on $\left(\mathbf{r}=\mathbf{r}_{i}\right), \boldsymbol{\Omega}_{i}$ the rotation vector associated to $i$ and $a$ the radius of the particle.

If we impose a perturbation $\delta v_{i}$ to the velocity $\mathbf{v}_{i}$ of the single particle $i$, the whole velocity field is modified by an amount $\delta \mathbf{v}$. Since equations (1) are linear, in a free medium at rest at infinity $\delta v$ would take the values given by the simple Stokes law $[6,7]$

$$
\delta \mathbf{v}_{\mathbf{s}}=\delta \mathbf{v}_{i}\left(\frac{3 a}{4 r}+\frac{a^{3}}{4 r^{3}}\right)+\mathbf{n}\left(\mathbf{n} \delta \mathbf{v}_{i}\right)\left(\frac{3 a}{4 r}-\frac{3 a^{3}}{4 r^{3}}\right)
$$

where

$$
\mathbf{n}=\frac{\mathbf{r}}{r}
$$

But the application of the non-slip boundary condition to the other spheres (and eventually to the other boundaries of the system) produces supplemental gradients which increase the dissipation and the apparent viscosity in the vicinity of $i$. Thus, the other particles act in some respect like an elastic and porous wall, and $\delta \mathbf{v}$ is modified. On these physical grounds we expect a decrease in the range of $\delta \mathbf{v}$. We shall refer to this as the "screening " (or " shielding ") effect. In flows past fixed (periodic or random) arrays of spheres, where the image of a porous wall (inelastic) is more evident, this effect is dominant at all concentrations and has been studied quantitatively in the dilute limit $[2,8]$ but we are interested in suspensions rather than in porous materials and the particles are supposed to be free to move. Of course, the exact shape of $\delta \mathbf{v}$ depends on the exact positions of the surrounding spheres. But we are only interested in statistical properties and look for a screening shape function applied to $\delta \mathbf{v}_{\mathbf{s}}$ to get the mean distribution $\delta \mathbf{v}_{r}$ in the same way as the Debye-Hückel theory of ionic solutions leads to a screening factor on the normal Coulomb potential.

The problems commonly studied in the theory of suspensions, such as the effective viscosity in homogeneous shear flows or the sedimentation velocity in homogeneous systems extending to infinity, do not seem to be well suited, both from the experimental and the theoretical points of view to the analysis of the pure screening effect, since in the former cases it is difficult to separate screening from larger global effects : for example in the sedimentation problem the main part of the decrease in the sedimentation velocity results, as clearly shown in references [1] and [5], from a backflow developed to balance the amount of fluid displaced upwards by the falling spheres. The screening should be involved only through corrective terms. The main purpose of this paper is to propose a very simple problem, which exhibits a pure screening effect and seems tractable both experimentally and theoretically. Consider an infinite homogeneous system of neutrally buoyant spheres. They are in equilibrium and $\mathbf{v}_{i}=0$. Now we add a single sphere of 
slightly higher density : under the action of gravity, it falls slowly in the homogeneous suspension with a mean constant velocity $\delta \mathbf{v}_{\mathrm{s}}$. The velocity field created in the suspension can be identified with a "shielded " field $\delta v$. Note that there is no restriction on the radius $a$ of this « test sphere " but the most interesting case is when $a$ is equal to the radius $a_{0}$ of the other spheres, since this is the physical realization of a single perturbation in a homogeneous neutrally buoyant suspension. Experimentally, such a system can be easily obtained by matching the density of the fluid (by preparing a liquid mixture of several constituents with the density of the spheres and by adding a single heavier particle). The motion of this " test sphere " generates around a cloud of dragged particles moving with the fluid. The size and shape of this cloud give some measurement of the interaction range and of the screening effect. The velocity $\mathbf{u}$ of the test particle is related to the total energy $E$ dissipated in the shielded velocity field by

$$
\mathbf{F u}=\frac{\mathrm{d} E}{\mathrm{~d} t}
$$

where $\mathbf{F}$ is the buoyancy force.

Although the screening studies are mainly intended to give some insight into the high concentration behaviour, there is some interest to try to predict the leading behaviour of the screening length $\xi$ in the limit of very dilute suspensions on the basis of very rough assumptions. In this case the screening length goes to infinity when the concentration goes to zero and the suspension behaves more or less like an effective fluid on distances large compared to the particle size. Note at first, that any attempt to introduce the screening effect through an a priori isotropic function of $r$ as would be, for example an exponential factor $\exp \left(-\frac{(r-a)}{\xi}\right)$ would be unsuccessful. The effective fluid would not obey the conservation laws. We shall rather try to express the velocity of the test particle in two different ways.

According to the Einstein law [6, 7] the apparent macroscopic viscosity of a dilute suspension, or "effective viscosity" is given by $\eta^{\prime}=\eta\left(1+\frac{5}{2} c+\mathcal{O}\left(c^{2}\right)\right)$ where $\eta$ is the viscosity of the pure fluid and $c$ the volume factor of the solid phase. For $a \gg a_{0}$ the test sphere can certainly be considered as moving in a fluid of higher viscosity $\eta^{\prime}$, and its velocity is reduced according to :

$$
u=u_{0}\left(1-\frac{5}{2} c+\cdots\right)
$$

where $u_{0}$ would be the velocity of the test sphere in an infinite medium. This result is supposed to be still a valid approximation which differs only by the precise numerical factor when $a$ is of the same order that $a_{0}$, if the suspension is dilute enough for the above conditions to hold.

It is more difficult to express $u$ as a function of $\xi$. The total energy dissipated by a sphere of velocity $u$ in an infinite pure fluid at rest at infinity would be :

$$
\frac{\mathrm{d} E}{\mathrm{~d} t}=-6 \pi \eta a u^{2} \quad\left(u=u_{0}, \text { Stokes law }\right) .
$$

In the suspension, for an equal velocity of the test sphere the dissipated energy is expected to increase, in relation with the appearance of a screening length, so that we may write :

$$
\frac{\mathrm{d} E}{\mathrm{~d} t}=-6 \pi \eta a u^{2}(1+\mathfrak{D}(\varepsilon))
$$

where $\varepsilon=a / \xi, \mathfrak{D}(\varepsilon)$ is a positive function and $\mathfrak{D}(\varepsilon) \rightarrow 0$ when $\varepsilon \rightarrow 0$ (i.e. $c \rightarrow 0$ ). From (2) we get immediately

$$
u=\frac{u_{0}}{1+\mathfrak{D}(\varepsilon)}
$$


and equating (3) and (4) with $c$ small we get

$$
D(\varepsilon) \approx \frac{5}{2} c
$$

but the leading term in $\mathfrak{D}(\varepsilon)$ is not known and must be induced from an additional hypothesis or evaluated by means of a model. If we assume now that the development of $\mathcal{D}(\varepsilon)$ is regular and the first linear term is non zero we find from (5) that $\xi \sim a / c$.

Some justification of such a linear behaviour can be found in the following rough model : we assume that the effect of the surrounding particles is fully represented by the screening length, so that the fluid still obeys the Navier-Stokes equations, but the flow is modified in such a way that the velocity comes close to zero at a distance $\xi$ of the test sphere. If we stipulate that this velocity is exactly zero, then the problem is equivalent to finding the velocity field of a fluid enclosed between an inner sphere of radius $a$, velocity $u$, and an outer concentric sphere of radius $\xi$, at rest. Such models have been used for the classical sedimentation problem (for a criticism, see Ref. [1]) in the framework of the so-called "cellular theories ". But the additional assumption $\xi=a c^{1 / 2}$ is generally done in order to equate the volume fraction of the inner sphere to the volume fraction $c$ of the suspension. It is well known (see Ref. [1]) that this leads to a wrong dependence of $u$ in $c^{1 / 3}$, in place of the correct behaviour in $c$. But our problem is completely different since external forces are applied only to the test sphere. Anyway, there is no physical reason to accept an a priori dependence of $\xi$ on $c$, since it represents the result of complex interactions and not a real physical barrier. This is precisely our purpose to determine such a relation and in our case the velocity has been already deduced in (3). From the general Stokes solution for flows with spherical symmetry [9] and given the above limit conditions, long and tedious, but rather straightforward calculations lead to an exact expression for the dissipated energy. Keeping only the leading term in $\varepsilon$ we get

$$
\mathscr{D}(\varepsilon)=\frac{9}{4}+\cdots \quad \text { or } \quad u=u_{0}\left(1-\frac{9}{4} \varepsilon+\cdots\right)
$$

(this last result exact to order 3 ) so that finally $\varepsilon=\frac{10}{9} c$ or $\xi \approx a / c$ with a numerical factor close to unity.

Due to the crudeness of the hypothesis this simple dependence of $\xi$ on $c$ is still questionable. Anyway, extension to the high concentration domain is not possible. In order to check this result, and to extend the studies of screening to these more interesting domains, two ways can be used :

- direct evaluation of the screening length from numerical solutions of the Navier-Stokes equations for clusters of size larger than $\xi$. This last condition restricts this approach to large concentrations ( $\xi$ small). Such a work is presently in progress;

- direct measurements on the experimental system described above. However, secondary effects might be considered. Due to the deformation of the flow lines the distribution of the particles around the test sphere could deviate from the initial distribution, assumed random, and gives a strictly correct description of screening only for this precise distribution (which besides provides a probably better approach to the general case in real suspensions). We also neglect the fluctuations of the velocity $u$ (the exactly fixed quantity is the buoyancy force) but this is in the spirit of a mean field theory for which we intend to develop the screening approach. For these two reasons this experimental approach seems accurate especially for moderate to medium concentrations.

To the end, we shall make two additional comments. First, the screening in suspensions of freely moving spheres is fundamentally different from the one observed in fixed arrays of spheres. In the latter case the other particles are fixed and act effectively as a porous wall $[8,10]$ whereas in the former case the spheres are transported with the fluid and the screening results mainly from additional gradients and dissipation around the particles. Except in the proposed experi- 
ment, chosen in order to eliminate all the other effects, it will normally appear only as a corrective term at low concentrations and will be of chief interest only for high concentrations.

Second, one must not confuse the screening length $\xi$ with the correlation length of the system. In a cluster approach of suspension rheology, for example, $\xi$ would be related to the coordination number rather than to the cluster size which represents the correlation length.

To conclude, we should like to emphasize again the interest of performing the study of the motion of a single falling sphere in a concentrated dispersion of neutrally buoyant freely moving spheres. It would be a good model for slow migration of isolated particles under external fields in such a medium, as well as a good situation to understand the screening properties of these mediums.

Acknowledgments. - We are very grateful to D. Stauffer for suggesting this work and supporting it with fruitful discussions and constructive criticism, to J. Kertész for valuable comments and to the Alexander von Humboldt Foundation for granting us a research fellowship.

\section{References}

[1] Batchelor, G. K., J. Fluid Mech. 52 (1972) 245.

[2] Childress, S., J. Chem. Phys. 56 (1972) 2527.

[3] Saffman, P. G., Studies in Applied Mathematics 52 (1973) 115.

[4] De Gennes, P. G., J. Physique 40 (1979) 783.

De Gennes, P. G., Conference on Physico-Chemical Hydrodynamics III, Madrid (1980).

[5] Stauffer, D. and Clavin, P., J. Physique-Lett. 42 (1981) L-353.

[6] Landau, L. and Lifshitz, E., Mécanique des Fluides, Ch. 2 (Ed. Mir, Moscou).

[7] BAtchelor, G. K., An Introduction to Fluid Mechanics, Ch. 4 (Cambridge University Press).

[8] TAM, C. K. W., J. Fluid Mech. 38 (1969) 537.

[9] LAMB, H., Hydrodynamics (Cambridge University Press) 1945, p. 603.

[10] Brickman, A. C., Appl. Sci. Res. A 1 (1947) 27. 